\title{
Mechanical intestinal obstruction in a young adult male with mucoviscidosis
}

\author{
J. R. GARNHAM ${ }^{1}$ AND T. R. G. CARTER \\ From St. Bartholomew's Hospital, London
}

EDITORIAL SYNOPSIS In mucoviscidosis survival to adult life is uncommon. This paper reports in detail the case of a young man surviving to the age of 21 years and illustrates a complication which may occur in such patients. Management is discussed.

Mucoviscidosis is genetically transmitted, probably as a recessive trait, and the frequency of homozygotes has been estimated as 1 in 2,000 live births (Roberts, 1959). About $10 \%$ of patients present in infancy with meconium ileus, but intestinal obstruction in older patients is uncommon, as most patients die young from pulmonary complications. With modern methods of treatment for such pulmonary disease the prognosis is much improved. Consequently this rare case is presented with a view to demonstrating the problems of dealing with a type of patient likely to become more common.

\section{CASE HISTORY}

The boy, R.B., took four months to regain his birth weight of $9 \frac{1}{2} \mathrm{lb}$., and his bowels were open up to 10 times a day, with bulky, fatty, offensive stools. By the age of 1 year he had had an attack of measles with prolapse of the rectum. At the age of 3 years he was diagnosed as a case of coeliac disease, and treated with a low-fat diet and vitamins. In the years that followed he was always small for his age, but quite fit, except for an attack of whoopingcough and two attacks of pneumonia. He ate no special foods and continued to pass frequent and offensive stools until he was admitted to St. Bartholomew's Hospital for the first time, aged 14 years, with acute abdominal pain, diagnosed as subacute obstruction. He was treated conservatively and, after investigation, a diagnosis of mucoviscidosis was made. There was no relevant family history. He was sent home on a normal diet with pancreatic extract and, despite repeated respiratory infections, he made satisfactory progress over the next six years.

After three days of severe abdominal pain and vomiting he was admitted to Epsom District Hospital in December 1960. At laparotomy rather more than half the small intestine was packed with solid masses which could not be milked down the bowel. The pancreas felt 'reasonably normal'. On opening the bowel to clear it, much semisolid material was obtained from the upper reaches but, in the terminal part, there was a complete obstruction due ${ }^{1}$ Now at The Metropolitan Hospital, Kingsland Road, London E.8 to the contents being firmly plastered on to the mucosal surface. The contents could only be peeled off with difficulty, leaving behind a perfectly dry mucous membrane. The bowel was divided above the obstruction and the proximal jejunum anastomosed to the transverse colon, while the distal end was brought out as a reverse ileostomy (Fig. 1).

Six weeks later he was transferred to St. Bartholomew's Hospital, where several attempts were made to clear the obstructed reversed ileostomy by wash-outs with various solutions, all of which proved unsatisfactory. A severe respiratory infection and a mild blind-loop syndrome delayed further surgery for 10 weeks. A caecostomy was performed to facilitate further lavage of the terminal ileum. Post-operatively the patient suffered two respiratory infections and also developed signs of a vitamin $K$ deficiency before the loop was partly cleared.

The first step towards restoring continuity of the gut was closure of the caecostomy, but the wound broke

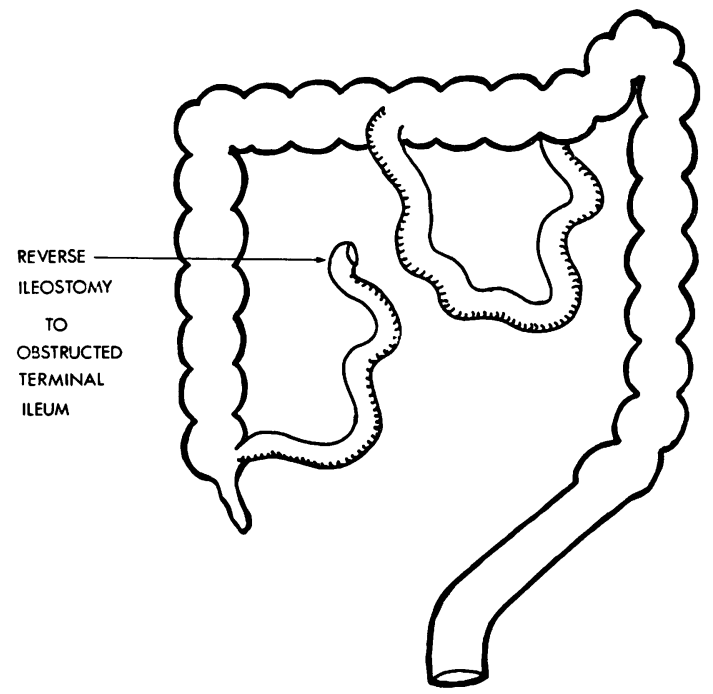

FIG. 1. Diagram of first-stage operation. 
down and the caecostomy prolapsed. He developed a diffuse bronchopneumonia and was sent home to avoid further cross infection in the ward.

On re-admission he complained of difficulty in seeing at night. A right upper homonymous visual field defect was discovered, along with some right-sided facial weakness. A cerebral abscess was suspected in view of the chronic pulmonary disease: investigation revealed nothing, and his vision returned to normal. A retrospective diagnosis of mild encephalitis, suppressed by the continuous antibiotic therapy, was made.

A further pulmonary infection preceded the eventual definitive operation of removal of the ascending colon and that part of the ileum which had originally been obstructed: by this stage some nine months had elapsed since his original admission for formation of the ileostomy.

The bowel was filled with viscid putty-like material. Post-operatively the condition of the chest steadily deteriorated and a resistant Proteus was isolated from the sputum. The heart suddenly failed, but the circulation was restored after open cardiac massage, and a tracheostomy was performed. He died the following day, 23 October 1961, at the age of 21 .

POST-MORTEM EXAMINATION The immediate cause of death was a diffuse bronchopneumonia. Besides such changes the lungs showed suppurative bronchiectasis (Fig. 2) and associated fibrosis. The pancreas was of normal size and shape, but felt rather firm, and floated in water (Fig. 3). Histology revealed a complete replacement of pancreatic parenchyma by adipose tissue, with normal islets of Langerhans (Fig. 4). The liver showed fatty change with evidence of early biliary cirrhosis, and

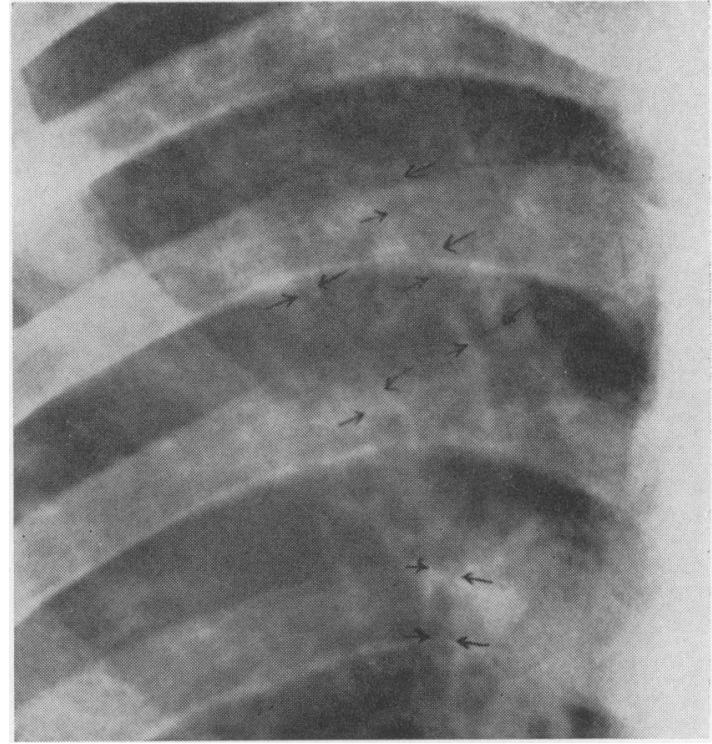

FIG. 2. Chest radiograph showing marked bronchiectasis.

the small gall bladder was full of very viscid mucus. A section of the wall of the gall bladder showed a prominent mucus-secreting epithelium, with distension of the glandular ducts and an excess of mucus. The duodenal walls presented a similar histological picture (Fig. 5). The remaining intestines were congested and bound together by dense adhesions but were not obstructed. There were no relevant findings elsewhere in the body.

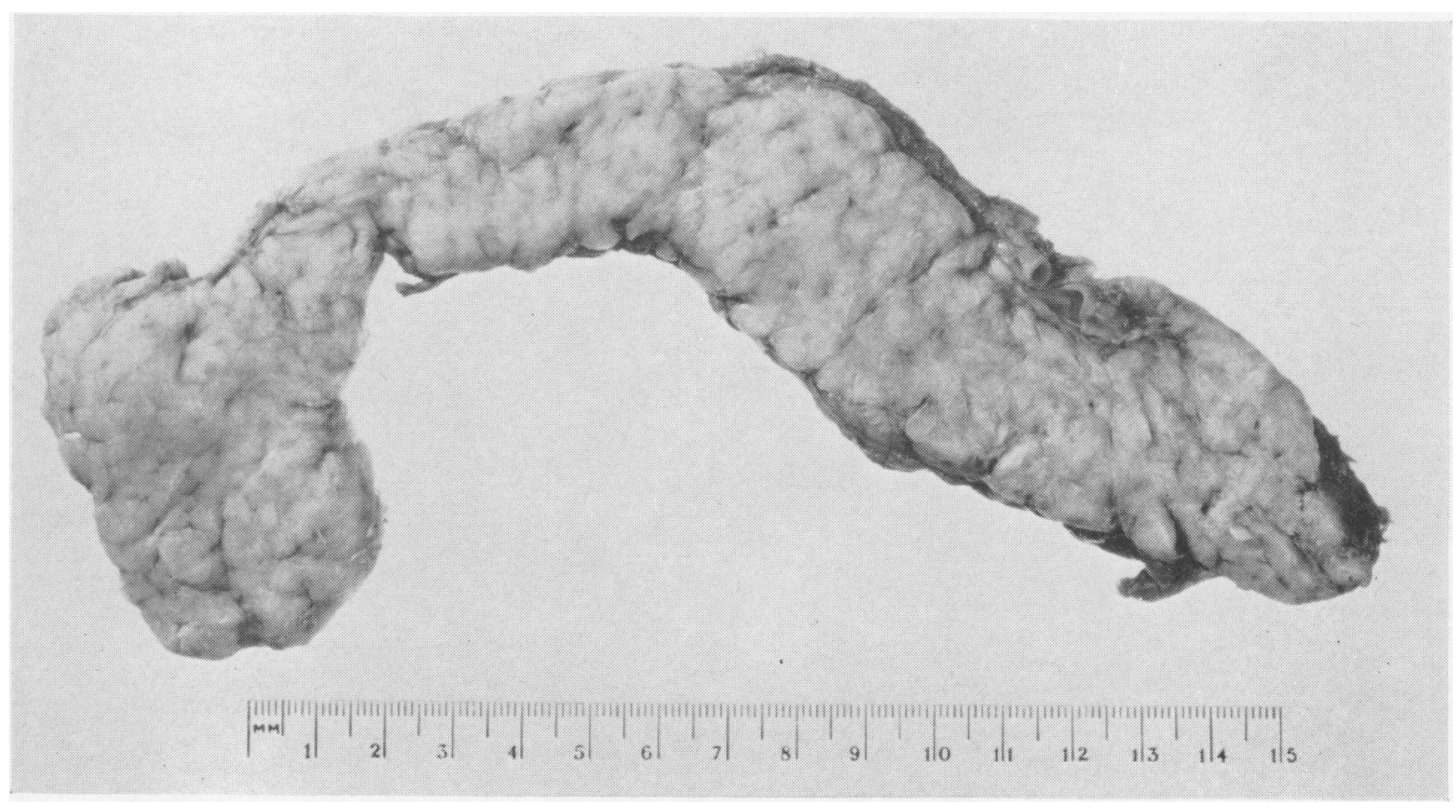

FIG. 3. The pancreas showing normal macroscopic appearances. 


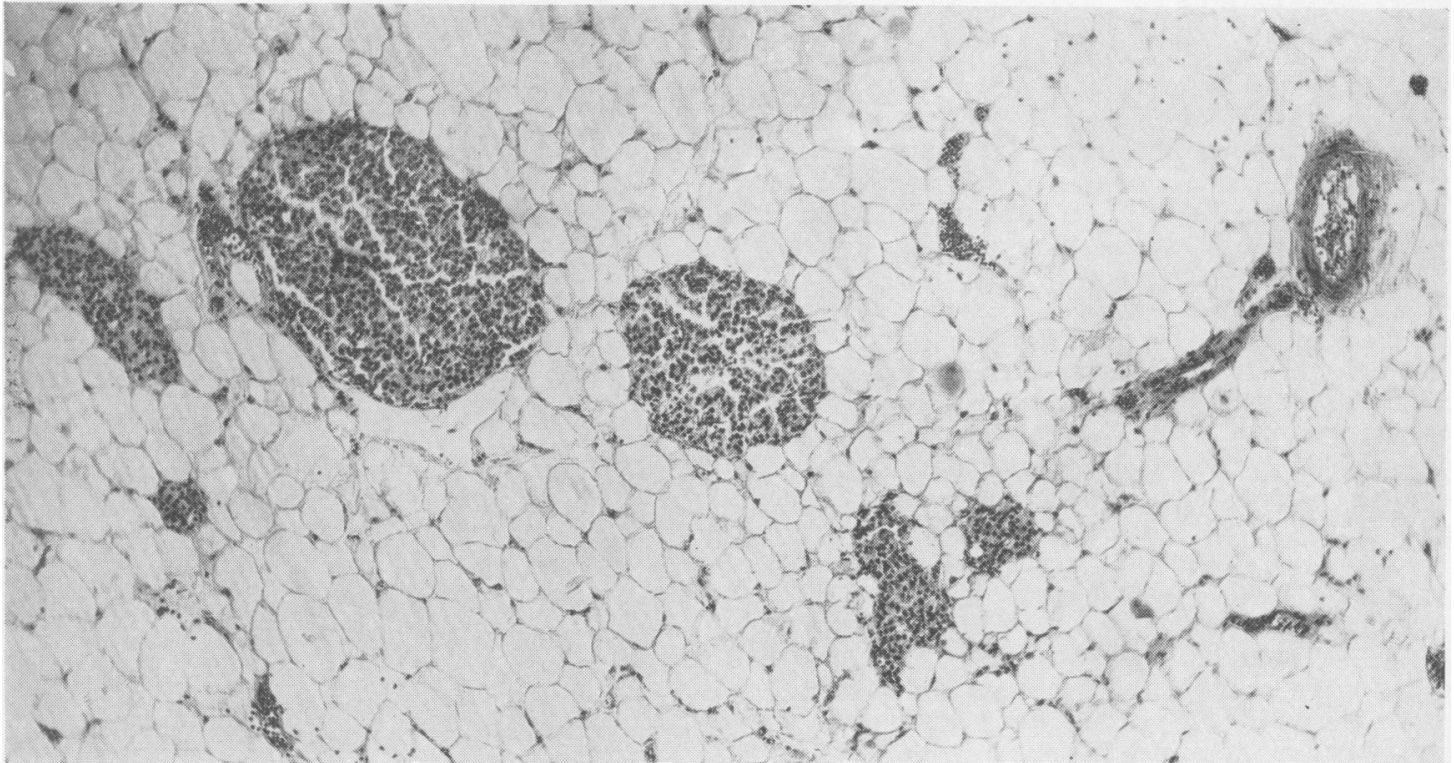

FIG. 4. Pancreas showing complete replacement of the acini by adipose tissue, leaving normal islets of Langherhans, vessels, and nerves.

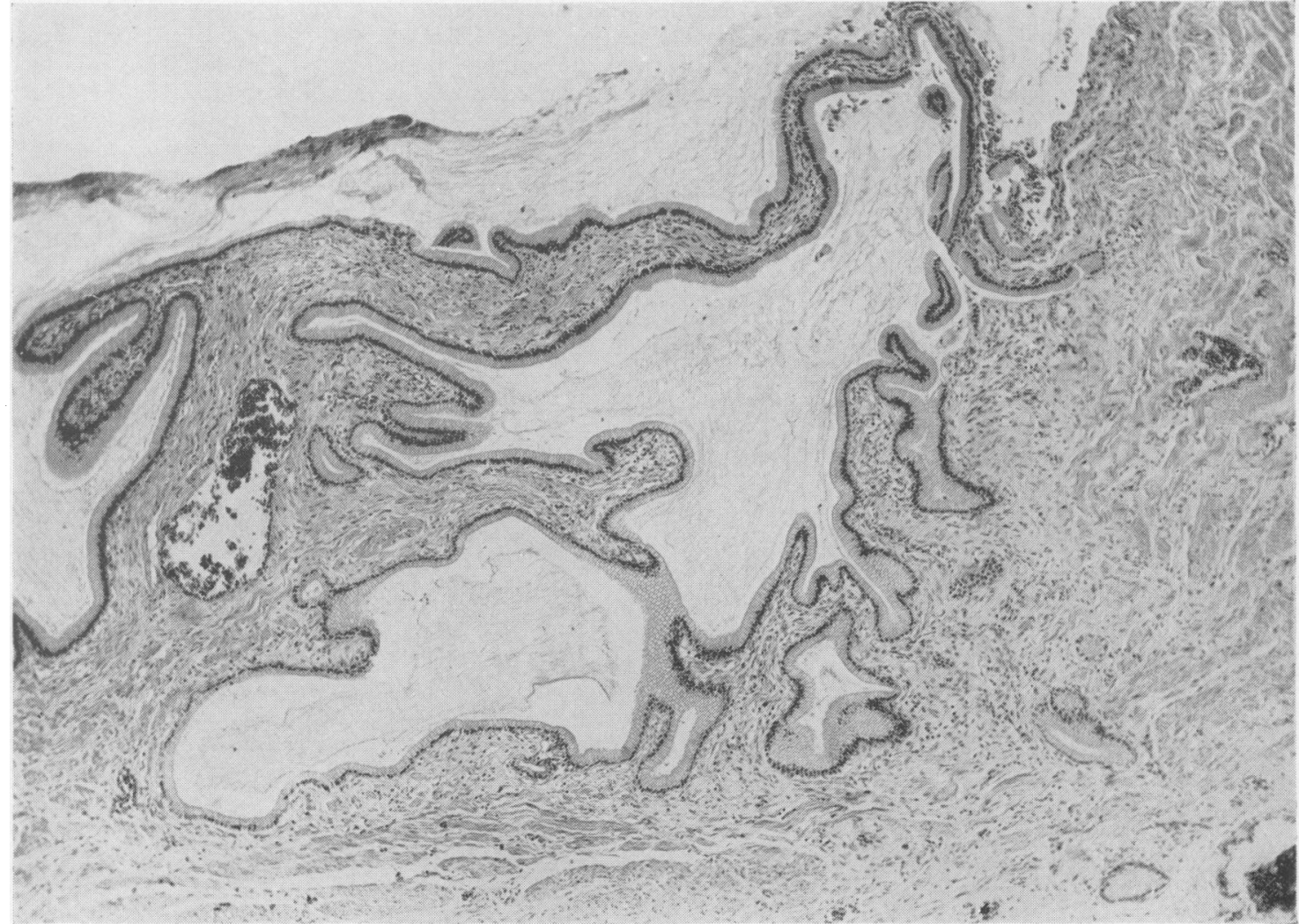

FIG. 5. Duodenal wall showing very prominent mucus-secreting epithelium and an excess of mucus with formation of Aschoff-Rokitansky sinuses. 


\section{DISCUSSION}

Survival to adolescence and adult life is rare in this condition. Marks and Anderson (1960) reported a case of mucoviscidosis in a man of 46 years. In 1959 (American Journal of Medicine) a case report appeared of a woman aged 17 who died of adult mucoviscidosis, the primary cause of death being pulmonary disease. Intestinal obstruction in this condition is most common in early infancy as meconium ileus. Hiatt and Wilson (1948) reported a series of 26 infants in all of whom approximately the distal half of the small intestine was filled with abnormal material. In the jejunum the contents were semi-liquid but gradually became nore dessicated in the distal loops. Porcaro and Soranno (1960) found in a boy aged 11 years with obstruction that there was thick tenacious material in the terminal $50 \mathrm{~cm}$. of the ileum. Yet again the terminal half of the small intestine was filled with semi-solid material.

The obstruction occurs at that part of the small intestine where the circular folds are deficient (Gray's Anatomy, 1949). This is the area where the intestinal contents are, in the normal person. most dehydrated, dehydration which is even more pronounced in mucoviscidosis, not so much as dehydration as such but due to the increased stickiness of the mucus. The terminal part of the bowel is less able to cope with such tenacious contents.

It may be argued that the infrequency of the obstruction in the adult is due to the increasing motility of the gut which occurs with age. The high incidence of overwhelming respiratory infection in these patients means that few survive to be subject to the abdominal complications.

In the series of infants operated upon by Hiatt and Wilson (op. cit.), the obstructed distal loop was washed out with saline. Farber (1944) reported a case in which pancreatin was instilled at operation through an enterostomy but death occurred later from intercurrent infection. Porcaro and Soranno (op. cit.) operated on an 11-year-old Negro boy and formed a distal ileostomy which they irrigated with pancreatin and $3 \%$ hydrogen peroxide: four weeks later they had to do an ileo-ascending colon anastomosis with resection of the distal ileum. Our patient had had a long and proven history of mucoviscidosis and over the previous years his most dominant symptoms had been respiratory. As in the case described by Porcaro and Soranno (op. cit.), subsequent events showed that conservative measures were inadequate and as a result he too had to undergo an ileo-colic anastomosis.

During the period in hospital necessary for a programme of restorative surgery, R.B. was exposed to the airborne flora of a general medical and surgical ward. In both of these places he developed exacerbations of the respiratory disease. Whereas in the normal environment of his home the infection could be kept at bay by the use of daily tetracyline therapy, in the artificial atmosphere of the ward this was not sufficient to thwart the organisms abounding in the air. While measures were in progress to clear the distal loop, R.B. developed a mild blind-loop syndrome, together with the complications of a transient encephalitis and vitamin K deficiency.

As yet there is no sure way of maintaining these patients in adequate nutritional balance, though with the availability of pancreatin and high-protein food supplements, and with the numerous antibiotics which can control the chronic respiratory infection, a more hopeful prognosis can be given.

This is a rare condition and the available case histories are few. Should one come across the same condition in an adolescent or young adult it would seem wise at first to try to clear the affected bowel with saline and detergent solutions. Mindful of the respiratory complications that may be made more prevalent by a long period in hospital, and the experience of two attempts at conservative treatment, we think that primary resection of the obstructed loop should be performed if clearance has not been possible.

Post-operatively, it would seem to be an advantage to nurse the patient in isolation, to give appropriate antibiotics for any infected sputum as is necessary, and to return the patient as soon as possible to his normal environment.

We are grateful to Mr. C. Naunton Morgan and Dr. E. R. Cullinan for permission to publish this case, to Dr. W. J. Hanbury for the post-mortem findings, to $\mathrm{Mr}$. Peter Crocker for the high-power photographs, and to the Department of Medical Photography, St. Bartholomew's Hospital, for the other photographs. One of us (J.R.G.) is grateful to his secretary, Mrs. Gillian Elkington, for considerable help in preparing the manuscript for publication.

\section{REFERENCES}

American Journal of Medicine (1959). 'Adult' mucoviscidosis. 27, 483-493.

Farber, S. (1944). Pancreatic function and disease in early life. V. Pathologic changes associated with pancreatic insufficiency. Arch. Path. 37, 238-250.

Gray's Anatomy (1949). Edited by T. B. Johnston and J. Whillis, 30th ed., p. 1374. Longmans, London.

Hiatt, R. B., and Wilson, P. E. (1948). Celiac syndrome. VII. Therapy of meconium ileus. Surg. Gynec. Obstet., 87, 317-327.

Marks, B. L., and Anderson, C. M. (1960). Fibrocystic disease of the pancreas in a man aged 46. Lancet, 1, 365-367.

Porcaro, F., and Soranno, C. A. (1960). Mechanical intestinal obstruction as a late complication of mucoviscidosis. Amer. J. Surg. 100, 113-116.

Roberts, J. A. F. (1959). An Introduction to Medical Genetics, 2nd ed. Oxford University Press, London. 